\title{
Influence of primary fragment excitation energy and spin distributions on fission observables
}

\author{
Olivier Litaize ${ }^{1, \star}$, Loïc Thulliez ${ }^{1,2}$, Olivier Serot ${ }^{1}$, Abdelaziz Chebboubi $^{1}$, and Pierre Tamagno ${ }^{1}$ \\ ${ }^{1}$ CEA, DEN, DER, SPRC, Cadarache, 13108 Saint-Paul-lez-Durance, France \\ ${ }^{2}$ CEA, Irfu, Université Paris-Saclay, 91191 Gif-sur-Yvette, France
}

\begin{abstract}
Fission observables in the case of ${ }^{252} \mathrm{Cf}(\mathrm{sf})$ are investigated by exploring several models involved in the excitation energy sharing and spin-parity assignment between primary fission fragments. In a first step the parameters used in the FIFRELIN Monte Carlo code "reference route" are presented: two parameters for the mass dependent temperature ratio law and two constant spin cut-off parameters for light and heavy fragment groups respectively. These parameters determine the initial fragment entry zone in excitation energy and spin-parity $\left(\mathrm{E}^{*}, \mathrm{~J}^{\pi}\right)$. They are chosen to reproduce the light and heavy average prompt neutron multiplicities. When these target observables are achieved all other fission observables can be predicted. We show here the influence of input parameters on the saw-tooth curve and we discuss the influence of a mass and energy-dependent spin cut-off model on gamma-rays related fission observables. The part of the model involving level densities, neutron transmission coefficients or photon strength functions remains unchanged.
\end{abstract}

\section{Introduction}

The primary fission fragment (FF) entry zone in excitation energy and spin-parity $\left(E^{*}, J^{\pi}\right)$ is one of the key points in the simulation of the fission process leading to prompt particle emission. Theory as well as experiments are not yet able to clearly identify these initial primary FF nuclear states. Models that are used in Monte Carlo codes allow to reproduce the neutron multiplicity or the gamma multiplicity but it is quite difficult to achieve both of them in one shot. Neutron and gamma multiplicities are the end point of the whole de-excitation process. Several models have to be used in order to calculate level densities, photon strength functions, optical model potential for neutron transmission coefficients. At low energies the nuclear level scheme, that is to say the knowledge of level energies, spins, parities, half-lives, intensities of the transitions from level to level have to be accounted for through experimental databases. In the FIFRELIN Monte Carlo code, the reference calculation route usually involves five input parameters (here one of them, the fraction of rigid body moment of inertia is fixed to 1.). They are chosen to calculate the initial states of primary fragments and reproduce a fission target observable such as the average prompt neutron multiplicity $\bar{v}$. We will show in this work how the main four parameters influence the average prompt neutron multiplicity as a function of pre-neutron fragment mass (the so-called saw-tooth). On the other hand we will discuss the influence of the spin cut-off parameter model used to assign the initial FF spin.

^e-mail: olivier.litaize@cea.fr 


\section{Quick reminder of the FIFRELIN model}

Pre-neutron fission fragment mass A and kinetic energy KE distributions are experimental data used as input data in the code. Data from [1] are used in this work for the study of ${ }^{252} \mathrm{Cf}(\mathrm{sf})$. Recent available data have been published [2] and will be used in a near future for comparison but this is out of the scope of this paper. Details related to the FIFRELIN "calculation route" can be found in [3] and [4]. The main steps are reminded hereafter.

Nuclear charge $\mathrm{Z}$ is sampled from the so-called Wahl $Z_{p}$ model. When the light fragment $A_{L}, Z_{L}$, $K E_{L}$ characteristics are sampled, the complementary heavy fragment of a binary fission is selected by mass, charge and linear momentum conservation. Before starting the de-excitation process, the excitation and spin-parity of both fragments have to be known. The total excitation energy TXE is calculated from the kinetic energy of both FF and the $Q$-value of the fission process knowing the binding energies of the fissioning nucleus and the light and heavy primary fragments. This intrinsic part of TXE is shared between the two fragments $E_{L}^{*}$ and $E_{H}^{*}$ through a $E^{*}=a T^{2}$ relation where $a$ stands for the level density parameter (Ignatyuk's prescription) and $T$ the temperature. Light and heavy FF temperatures are linked together through a mass-dependent temperature ratio law [3]. Before that, the rotational energy, considering a simple rotating liquid drop, has been subtracted from TXE. The calculation of the rotational energy requires a moment of inertia and a total angular momentum which is spin cut-off model-dependent.

$$
T X E=E_{L}^{*}+E_{H}^{*}+E_{L}^{r o t}+E_{H}^{r o t}
$$

where $E_{L}^{r o t}, E_{H}^{r o t}$ are the rotational energies of the light and heavy fragments and $E_{L}^{*}, E_{H}^{*}$ are their intrinsic excitation energies. The rotational energy $E^{r o t}$ is simply expressed considering a rotating liquid drop:

$$
E^{r o t}=\frac{\hbar^{2} J(J+1)}{2 I}
$$

where $J$ stands for the total angular momentum sampled from Eq. (3) following Bethe's work.

$$
P(J)=\frac{J+1 / 2}{\sigma^{2}} \exp \left(-\frac{(J+1 / 2)^{2}}{2 \sigma^{2}}\right)
$$

The factor $\sigma^{2}$ is the spin cut-off parameter which can be constant or energy-dependent and will be studied in section 4 . The nuclear moment of inertia $\mathcal{I}$ is defined as a proportion $k_{\text {rig }}$ of a rigid spheroid $I_{\text {rig }}$ defined by:

$$
\mathcal{I}_{\text {rig }}=\frac{2}{5} A M R^{2}\left(1+0.31 \beta_{2}+0.44 \beta_{2}^{2}+\cdots\right)
$$

where $A, M, R$ and $\beta_{2}$ respectively stand for the mass number, the nucleon mass, the radius $(R=$ $1.2 A^{1 / 3} \mathrm{fm}$ ) and the quadrupole deformation parameter of the nucleus in its ground state taken from a Myers-Swiatecki parameterization of the mass formula. The total amount of excitation energy is deduced from the energy release during the fission process $Q$ based on binding energies from [5] and the sampled total kinetic energy:

$$
T X E=Q-T K E
$$

Here we consider the spontaneous fission of ${ }^{252} \mathrm{Cf}$. Only the intrinsic excitation energy $E^{*}$ corresponding to $T X E-E^{r o t}$ is treated within a Fermi gas approximation in $a T^{2}$ where $a$ and $T$ stand for the level density parameter and the nuclear temperature. An iterative procedure is used to determine 


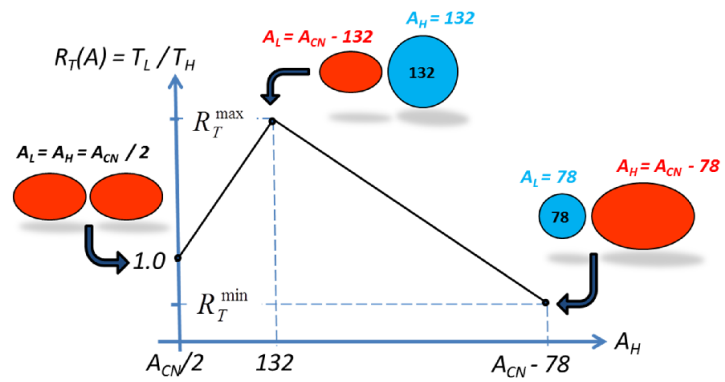

Figure 1. Temperature ratio law $R_{T}(A)$ : three anchor points are considered from macroscopic nucleus shapes at scission to determine the temperature ratio of two complementary fission fragments after full acceleration when they have recovered their ground state deformation (see text for details).

the fragment excitation energy because the level density parameter is energy/temperature dependent following the Ignatyuk prescription:

$$
a=\bar{a}\left\{1+\frac{\delta W}{U^{*}}\left(1-e^{-\gamma U^{*}}\right)\right\}
$$

where $\bar{a}(A)$ is the asymptotic level density parameter, $\delta W$ accounts for the shell corrections that can be estimated by the difference between measured and calculated mass excesses (with a liquid drop model for example), and $U^{*}=E^{*}-\Delta$ is an effective excitation energy function corrected by pairing $\Delta$ while $\gamma$ is a damping factor. Several implementations of these parameters are driven through the RIPL-3 documentation [6].

For a given fragmentation, the temperature of the light fragment $T_{L}=T\left(A_{L}\right)$ is linked to the temperature of its heavy partner $T_{H}=T\left(A_{H}\right)$ by a mass dependent temperature ratio law $R_{T}(A)=$ $T_{L} / T_{H}$ (Fig. 1). This law has been already described elsewhere [3]. Briefly the maximum of this law $\left(R_{T}^{\max }\right)$ is supposed to correspond to a minimum temperature of the heavy fragment which is linked to a minimum in the deformation energy (minimum in the excitation energy). This minimum occurs for the doubly closed spherical shell nuclei $(\mathrm{Z}=50, \mathrm{~N}=82$ and $\mathrm{A}=132)$. The situation is completely reversed with a minimum in the temperature ratio $\left(R_{T}^{m i n}\right)$ when the light fragment has a proton and a neutron closed spherical shell $(\mathrm{Z}=28, \mathrm{~N}=50$ and $\mathrm{A}=78)$. Finally for symmetric fission, the temperature of the two identical complementary fragments is the same, the ratio is equal to one. In between these anchor points, the evolution is supposed to be linear. Once this law is established, all the ingredients are known and can be used to calculate the excitation energies of the two complementary fragments through the iterative scheme:

$$
\left\{\begin{aligned}
E_{L}^{*} & =\frac{T X E-E^{r o t}}{1+\frac{a_{H}}{a_{L} R_{T}^{2}}} \\
E_{H}^{*} & =\frac{T X E-E^{\text {rot }}}{1+\frac{a_{L} R_{T}^{2}}{a_{H}}}
\end{aligned}\right.
$$

At this stage, the characteristics $\left(A, Z, K E, E^{*}, J, \pi\right)$ of the fission fragments are completely known and the deexcitation process can start by neutron, gamma and conversion electron emission from primary excited fragments (Fig. 2).

The statistical de-excitation process in an energy, spin, parity ensemble, often called HauserFeshbach formalism, has been implemented in FIFRELIN code using the notion of nuclear realization first introduced for gamma emission [7] and extended to coupled neutron/gamma emission [8]. 
Figure 3 shows an example of two possible cascades with or without neutron emission. Neutron emission is taken into account by pre-tabulated neutron-transmission coefficients at the very beginning stage of the de-excitation. Gamma emission requires the calculation of photon strength functions and level densities from unknown levels to other accessible levels. Experimental intensities are used at low energies when nuclear structure is known. Conversion electron are also accounted for through conversion coefficients.

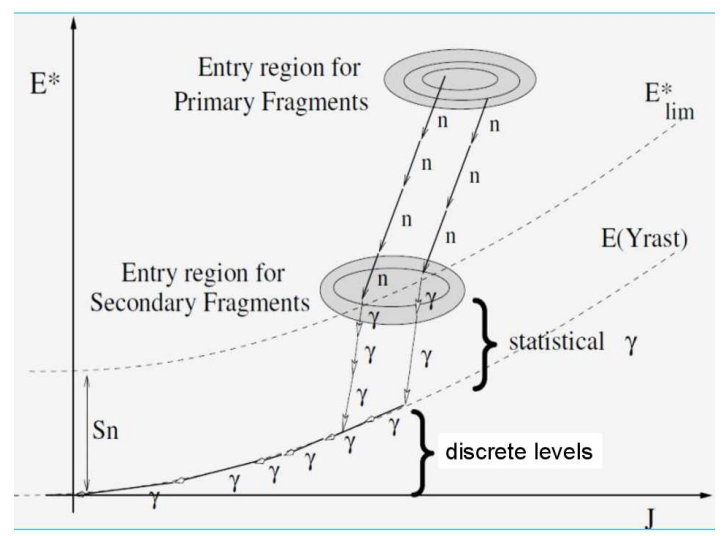

Figure 2. Schematic representation of FF de-excitation in a $\left(E^{*}, J\right)$ plan.

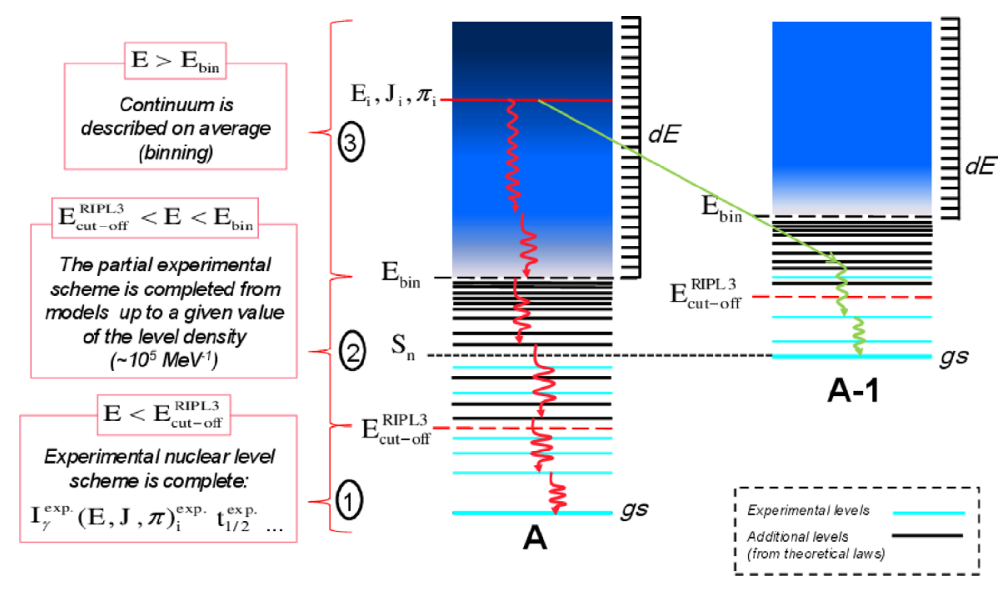

Figure 3. Nuclear de-excitation scheme in FIFRELIN.

\section{Influence of the free input parameters}

The four free input parameters of the reference calculation route are listed in the first line of Table 1 and reported hereafter:

$$
\left\{R_{T}^{\min } / R_{T}^{\max } / \sigma_{L} / \sigma_{H}\right\}=\{0.35 / 1.40 / 11.0 / 10.5\}
$$


Table 1. Influence of the free parameters on the neutron multiplicities and gamma multiplicities (without threshold energy and with a $3 n s$ coincidence time window). Statistical uncertainties are about 0.002 for neutron and 0.01 for gamma multiplicities.

\begin{tabular}{l|rrr|rrr}
\hline Input parameters & \multicolumn{3}{|c|}{ Neutron multiplicities } & \multicolumn{3}{|c}{ Gamma multiplicities } \\
$R_{T}^{\min } ; R_{T}^{\max } ; \bar{\sigma}_{L} ; \bar{\sigma}_{H}$ & $\bar{v}_{L}$ & $\bar{v}_{H}$ & $\bar{v}$ & $\bar{M}_{\gamma, L}$ & $\bar{M}_{\gamma, H}$ & $\bar{M}_{\gamma}$ \\
\hline $0.35 ; 1.4 ; 11.0 ; 10.5$ & 2.07 & 1.68 & 3.75 & 6.45 & 5.13 & 11.58 \\
$\mathbf{0 . 6 5} ; 1.4 ; 11.0 ; 10.5$ & 2.19 & 1.55 & 3.74 & 6.40 & 5.17 & 11.57 \\
$0.35 ; \mathbf{1 . 1} ; 11.0 ; 10.5$ & 1.71 & 2.08 & 3.79 & 6.55 & 5.00 & 11.55 \\
$0.35 ; 1.4 ; \mathbf{0 8 . 0} ; 10.5$ & 2.08 & 1.79 & 3.87 & 4.89 & 5.09 & 9.98 \\
$0.35 ; 1.4 ; 11.0 ; \mathbf{0 8 . 5}$ & 2.09 & 1.73 & 3.82 & 6.46 & 4.15 & 10.6 \\
\hline
\end{tabular}

This set of parameters allows to reproduce the total average prompt neutron multiplicities of the ${ }^{252} \mathrm{Cf}(\mathrm{sf})$ reaction $\bar{v}_{L}=2.07, \bar{v}_{H}=1.68$ and $\bar{v}=3.75$ compared to the experimental reference values (target observables) taken from Vorobyev [9]: $\bar{v}_{L}=2.05, \bar{v}_{H}=1.70$ and $\bar{v}=3.76$. The average multiplicity as a function of mass can also be observed in Fig. 4 compared with experimental data.

The influence of the free input parameters is studied from different variations of these parameters. The rest of the model remain unchanged:

- Composite Gilbert-Cameron model for level densities [10],

- AME-2012 for binding energies [5],

- RIPL3-2015 for nuclear level schemes [11],

- Koning-Delaroche optical model potential for neutron transmission coefficients [12],

- Enhanced Generalized Lorentzian model for E1 photon strength functions while additional XLtypes are accounted for using RIPL-3 recommendations [6].

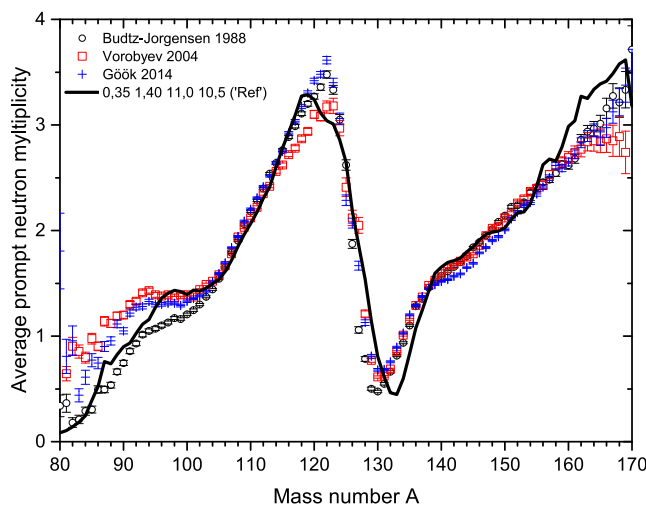

Figure 4. Average prompt neutron multiplicity as a function of pre-neutron fragment mass. Symbols are experimental data from $[2,13,14]$. Black line corresponds to the reference calculation.

A change in the $R_{T}$ law greatly affects the saw-tooth shape of the average prompt neutron multiplicity as a function of pre-neutron fission fragment mass (Fig. 5) and then the values for light and heavy 


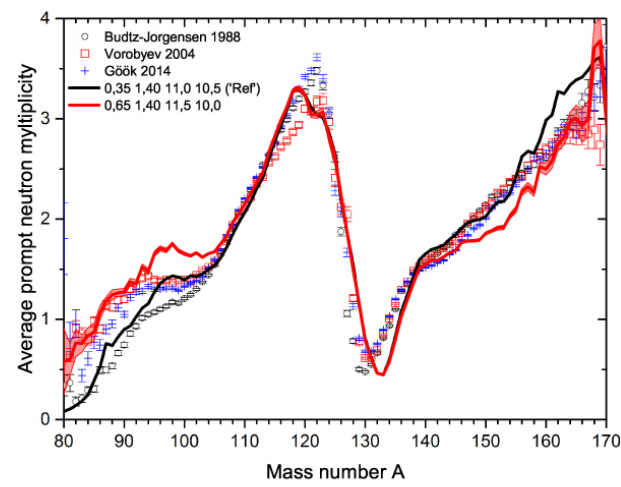

(a) $R_{T}^{\min }$ increases from 0.35 to 0.65

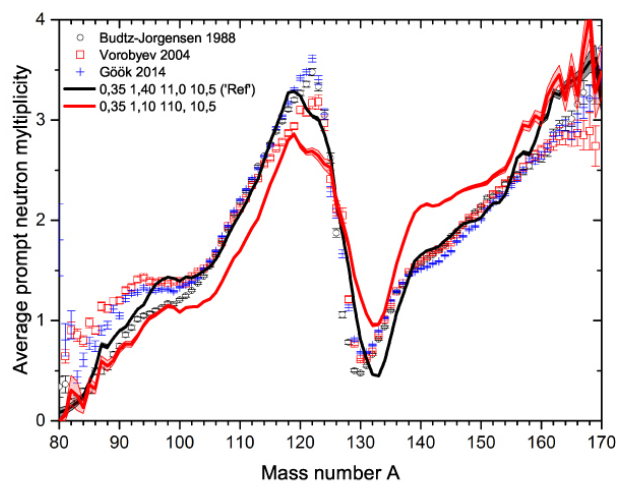

(b) $R_{T}^{\max }$ decreases from 1.40 to 1.10

Figure 5. Average prompt neutron multiplicity as a function of pre-neutron fragment mass. Symbols are experimental data. The influence of a modification of the $R_{T}(A)$ law is shown as red line compared to the reference calculation as black line.

groups $\bar{v}_{L}$ and $\bar{v}_{H}$. Due to a compensation between light and heavy groups, this does not influence so much the total average prompt neutron multiplicity $\bar{v}$ as it can be seen in Table 1 (second and third lines compared to first one).

Because $R_{T}=T_{L} / T_{H}$, an increase of $R_{T}$ (for instance $R_{T}^{\min }$ goes from 0.35 to 0.65 ) leads to a higher excitation energy for light fragments and a lower excitation energy for heavy fragments. Consequently the average prompt neutron multiplicity increases for light fragments and decreases for heavy fragments (second line of Table 1 and Fig. 5a). In the same way a decrease of $R_{T}$ (for instance $R_{T}^{\max }$ goes from 1.40 to 1.10 ) leads to a lower excitation energy for light fragments and a higher excitation energy for heavy fragments. Consequently the average prompt neutron multiplicity decreases for light fragments and increases for heavy fragments (third line of Table 1 and Fig. 5b).

The corresponding average gamma multiplicity is roughly $\overline{M_{\gamma}} \sim 11.6$ without gamma energy threshold and with a time coincidence window of about $3 \mathrm{~ns}$. With a $100 \mathrm{keV}$ threshold, this multiplicity is reduced to about $\overline{M_{\gamma}} \sim 10.6$. Compared to the neutron multiplicity, the gamma multiplicity is less sensitive to the variations of $R_{T}(A)$ because the very most part of gammas are emitted after neutrons cooling the fragment down to more or less the same nuclear state.

A change of initial spin cut-off parameters affects in a different way the saw-tooth shape of $\bar{v}(A)$. The two constant values for the spin cut-off modify the initial spin of primary fission fragments. This does not only affect the position of entry states along the $\mathrm{J}$ axis of Fig. 2 but also along the $E^{*}$ axis because $J$ is used to calculate the total rotational energy (see Eq. (2)). This is why a lower spin cut-off parameter for light fragments as well as for heavy fragments leads to an increase of the average total prompt neutron multiplicity. It is noticeable that it is specifically the neutron multiplicity for heavy fragment group that increases significantly compared to the light fragment group (fourth and fifth lines compared to first line in Table 1). The average gamma multiplicity is closer linked to this spin cut-off modification: if $\sigma_{L}\left(\sigma_{H}\right)$ decreases then the gamma multiplicity for light (heavy) group decreases.

\section{Influence of the spin cut-off model}

We have seen in the previous section that two different constant values can be used for the spin cut-off parameters of primary light and heavy fragment groups in order to reproduce $\bar{v}_{L}$ and $\bar{v}_{H}$ observables. 


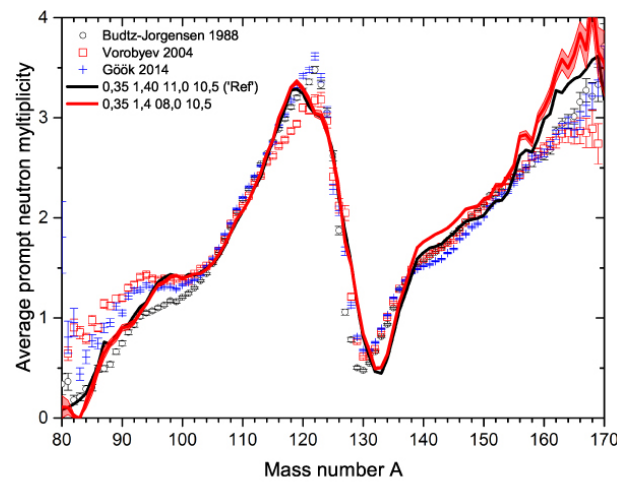

(a) $\bar{\sigma}_{L}$ decreases from $11 \hbar$ to $8 \hbar$

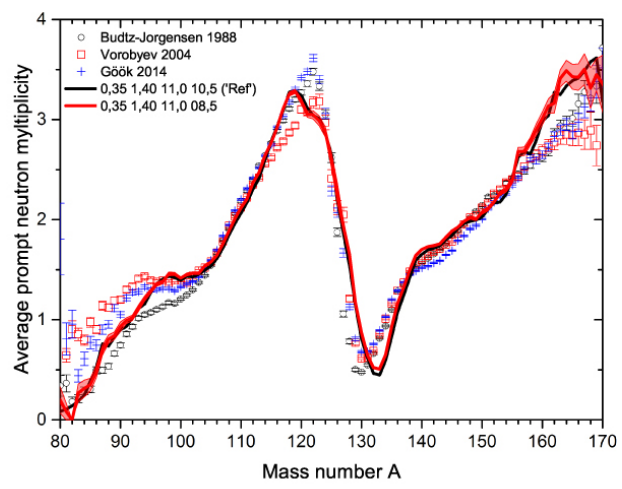

(b) $\bar{\sigma}_{H}$ decreases from $10.5 \hbar$ to $8.5 \hbar$

Figure 6. Average prompt neutron multiplicity as a function of pre-neutron fragment mass. Symbols are experimental data. The influence of a modification of the spin cut-off parameters is shown as red line compared to the reference calculation as black line.
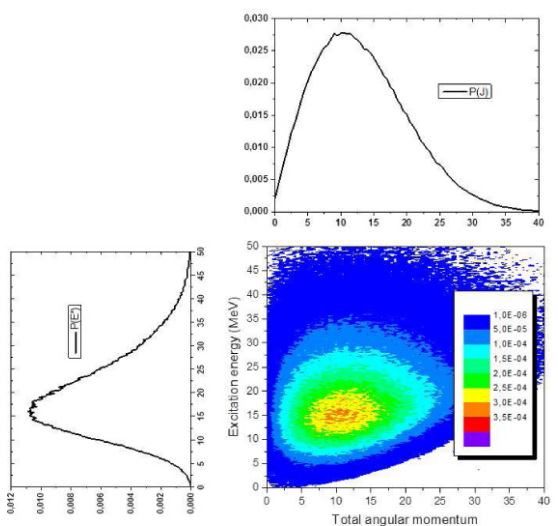

(a) Initial $\left(E^{*}, J\right)$ distribution for light fragments
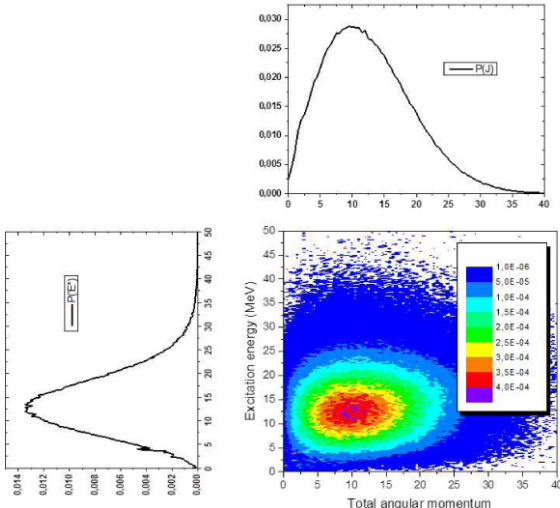

(b) Initial $\left(E^{*}, J\right)$ distribution for heavy fragments

Figure 7. Density of initial fission fragment states with constant spin cut-off parameter.

We can also consider an energy dependent spin cut-off model for sampling the initial total angular momenta (Inertia+ Shell model following preliminary calculations reported in [15]):

$$
\sigma^{2}=I_{\bar{a}}^{a} \sqrt{\frac{U}{a}}
$$

where $\mathcal{I}$ stands for the nuclear moment of inertia. This formula accounts for the energy dependence of the spin cut-off parameter as well as the shell effects through the additional term $a / \bar{a}$ where $a$ and $\bar{a}$ stand for the level density parameters of Eq. (6). The spin cut-off parameter is used to sample $J$ (Eq. (3)) and calculate $E^{r o t}$ in order to subtract from the total excitation energy. Here, the parameter $\sigma^{2}$ depends also on the excitation energy (which is not known yet at the beginning of the iterative 

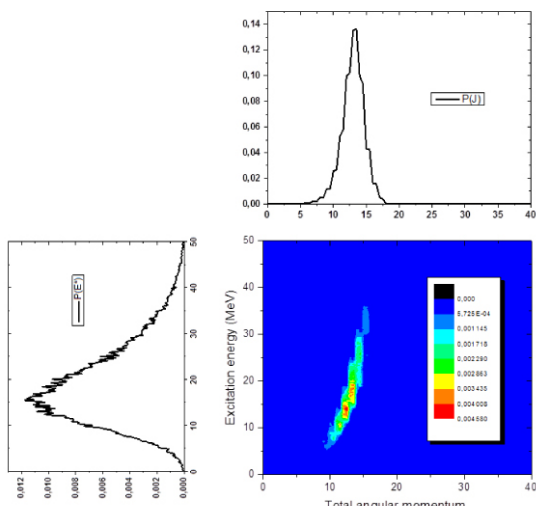

(a) Initial $\left(E^{*}, J\right)$ distribution for light fragments
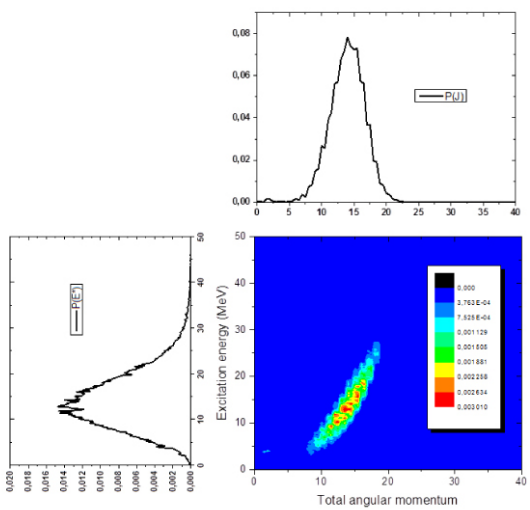

(b) Initial $\left(E^{*}, J\right)$ distribution for heavy fragments

Figure 8. Density of initial fission fragment states (pre-neutron emission) with an energy-dependent spin cut-off parameter.

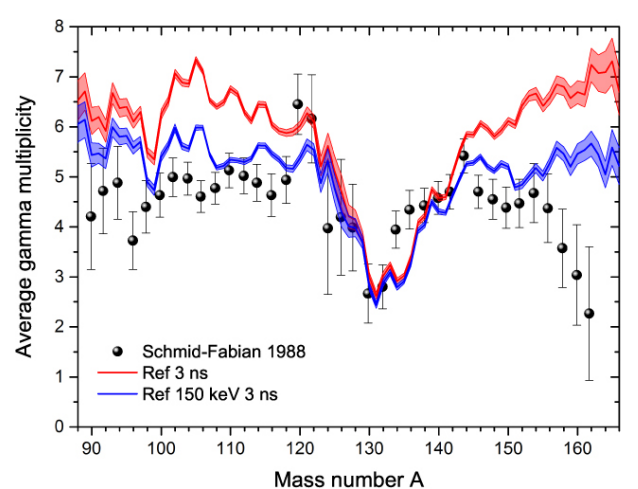

(a) Reference route

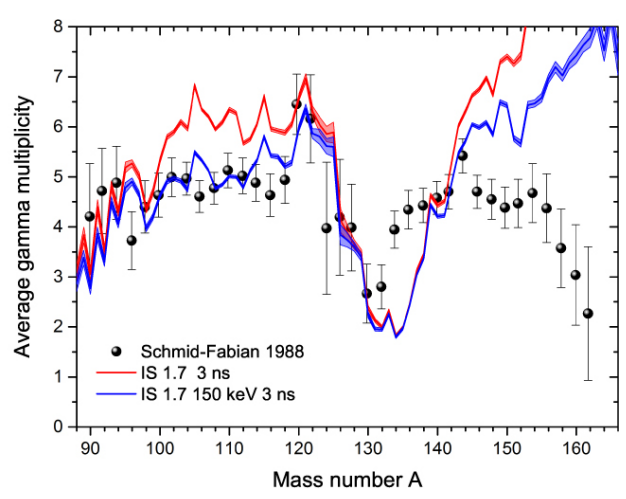

(b) Inertia+shell model

Figure 9. Average prompt fission gamma multiplicity as a function of mass for the ${ }^{252} \mathrm{Cf}(\mathrm{sf})$ reaction.

procedure used to solve Eq. (7)). We have considered in this work the average value of $J$ which is proportional to $\sigma^{2}$. The $\left(E^{*}, J\right)$ entry zone for primary fragments is shown in Fig. 7 using two constant spin cut-off values for light and heavy groups compared to the entry zone using an energy dependent spin cut-off model in Fig. 8.

A saw-tooth shape is more visible in the average gamma multiplicity as a function of pre-neutron mass when the mass- and energy-dependent spin cut-off model is used instead of constant values for light and heavy groups (Fig. 9). The gamma multiplicity is better reproduced for light fragments but the disagreement still persists for heavy fragments.

The gamma multiplicity distribution is directly linked to the primary spin distribution as we can see in Figs. 7 and 8 (the top part of figures corresponds to a X-projection in the $\left(E^{*}, J\right)$ distribution) compared to Fig. 10. 


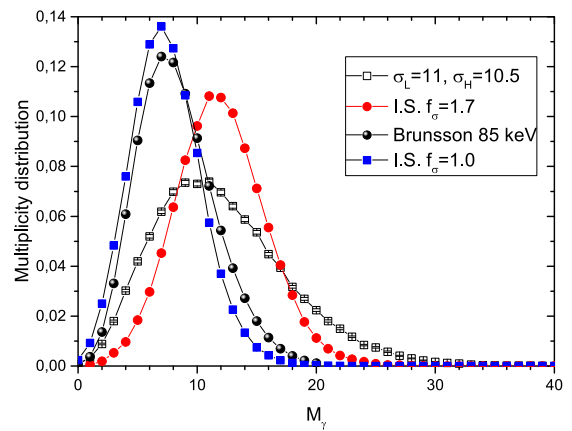

Figure 10. Prompt gamma multiplicity distribution. I.S. stands for Inertia+Shell spin cut-off model.

By using two constant spin cut-off values for primary FF (leading to good neutron multiplicity values) the average gamma multiplicity distribution is far from the double Poisson model used by Brunsson [16] to interpret experimental data from Pleasonton (the threshold of $85 \mathrm{keV}$ explains a part but not the whole effect). If we consider a inertia + shell model (I.S.) without re-scaling $\left(f_{\sigma}\right)$ the spin cut-off (and the level density) then the calculated gamma multiplicity distribution is clearly improved but the neutron average multiplicity is not reproduced. To reproduce the average neutron multiplicity (even as a function of mass) it is necessary to re-scale the spin cut-off by a factor $f_{\sigma}=1.7$ but then the gamma multiplicity distribution is degraded (Fig. 10). The corresponding average value is quite similar to the one obtained with constant spin cut-off values but the distribution is modified as expected.

\section{Conclusion}

The influence of the free input parameters used in the FIFRELIN code to estimate the primary excitation energy and spin distributions for the calculation of fission observables was studied as well as two different spin cut-off models used for sampling primary fission fragment spins. The present calculation routes are not able to reproduce both neutron and gamma multiplicities in a single run for ${ }^{252} \mathrm{Cf}(\mathrm{sf})$ suggesting that other ingredients of the model have to be improved such as level densities, neutron and photon transmission coefficients through optical models and strength functions respectively.

\section{References}

[1] N. Varapai, F.-J. Hambsch, S. Oberstedt, O. Serot, G. Barreau, N. Kornilov, S. Zeinalov, AIP Conf. Proc. 798, 369 (2005)

[2] A. Göök, F.-J. Hambsch and M. Vidali, Phys. Rev. C 90, 064611 (2014)

[3] O. Litaize, O. Serot, Phys. Rev. C 82, 054616 (2010)

[4] O. Litaize, O. Serot and L. Berge, The European Physical Journal A 51, 177 (2015)

[5] G. Audi, M. Wang, A.H. Wapstra, F.G. Kondev, M. MacCormick and X. Xu, Nuclear Data Sheets 120, 1 (2014)

[6] R. Capote et al., Nuclear Data Sheets 110, 3107 (2009) 
[7] F. Becvar, Nuclear Instruments and Methods in Physics Research Section A: Accelerators, Spectrometers, Detectors and Associated Equipment, 417 (1988)

[8] D. Regnier, O. Litaize and O. Serot, Computer Physics Communications 201, 19 (2016)

[9] A.S. Vorobyev, O.A. Shcherbakov, A.M. Gagarski, G.V. Val'ski and G.A. Petrov, EPJ Web of Conferences 118, 03004 (2010)

[10] A. Gilbert and A.G.W. Cameron, Canadian Journal of Physics 43, 1446 (1965)

[11] M. Verpelli, R. Capote, Report-INDC(NDS)-0702 (2015)

[12] A.J. Koning and J.P. Delaroche, Nuclear Physics A 713, 231 (2003)

[13] C. Budtz-Jorgensen and H.-H. Knitter, Nuclear Physics A 490, 307 (1988)

[14] A. Vorobyev, V. Dushin, F.-J. Hambsch, V. Jakovlev, V. Kalinin, A. Laptev, B. Petrov, O. Shcherbakov, AIP Conf. Proc. ND 2004 769, 613-616 (2005)

[15] L. Thulliez, O. Litaize, O. Serot, EPJ Web of Conferences 111, 10003 (2016)

[16] G.S. Brunson, Los Alamos Report LA-9408-T (1982) 